\title{
Causa natalium ad forum ecclesiasticum spectat :un pouvoir redoutable et redouté
}

\section{Anne Lefebvre-Teillard}

\section{(2) OpenEdition}

1 Journals

Édition électronique

URL : https://journals.openedition.org/crm/883

DOI : $10.4000 / \mathrm{crm} .883$

ISSN : 1955-2424

Éditeur

Honoré Champion

Édition imprimée

Date de publication : 15 février 2000

ISSN : 1272-9752

\section{Référence électronique}

Anne Lefebvre-Teillard, "Causa natalium ad forum ecclesiasticum spectat :un pouvoir redoutable et redouté », Cahiers de recherches médiévales [En ligne], 7 | 2000, mis en ligne le 03 janvier 2007, consulté le 15 décembre 2022. URL : http://journals.openedition.org/crm/883 ; DOI : https://doi.org/10.4000/ crm.883

Ce document a été généré automatiquement le 15 décembre 2022.

Tous droits réservés 


\title{
Causa natalium ad forum ecclesiasticum spectat :un pouvoir redoutable et redouté
}

\author{
Anne Lefebvre-Teillard
}

Causa natalium... ad forum ecclesiasticum spectat. Lorsqu'en 1223 le pape Honorius III, écrivant au roi de France, affirme ainsi la compétence de l'Église, personne n'en doute plus, à commencer par Louis VIII lui-même ${ }^{1}$. Il lui suffisait de se remémorer, en la matière, l'édifiant exemple de son père. En ce début du XIII ${ }^{e}$ siècle, c'est à l'Église qu'il appartient en effet de dire qui est légitime ou qui ne l'est pas. Pouvoir redoutable car de la qualité de légitime dépend celle d'héritier... pouvoir redouté pour cette raison même. C'est pourquoi tout en étant reconnu, il va être au cœur de vives controverses, voire, comme en Angleterre, d'affrontements entre les deux pouvoirs. L'enjeu était trop important pour un pouvoir temporel reposant essentiellement sur une transmission héréditaire, pour qu'il ne cherche pas, par différents moyens, à limiter, voire à éliminer la compétence de l'Église en cette matière. Une compétence que nous examinerons avant de voir quels furent les moyens mis en œuvre à son encontre.

2 La compétence de l'Église en matière de filiation légitime est étroitement liée à sa compétence devenue exclusive en matière de mariage: juger de l'existence ou de la validité d'un mariage dépend de l'Église et d'elle seule ${ }^{2}$. Or comme le montrent les décrétales d'Alexandre III (1159-1181) et d'Innocent III (1198-1216) réunies sous le titre Qui filii sint legitimi dans le recueil officiel des Décrétales de Grégoire IX (1234), la plupart, pour ne pas dire la quasi totalité, des contestations en légitimité sont fondées, à cette époque, sur l'inexistence ou l'invalidité du mariage des parents ${ }^{3}$. C'est donc par voie de conséquence que l'Église a été amenée à juger de la légitimité d'une personne. Elle le fait généralement de manière préjudicielle, c'est-à-dire avant toute décision sur le fond du procès, dans le cadre d'affaires successorales qui sont, elles, portées devant le juge séculier. Mais ses tribunaux peuvent aussi être directement saisis, à titre principal, de la question. 

Grégorienne avait redonné vigueur ${ }^{4}$. La réponse était apportée sous la forme d'une décrétale dans laquelle le pape, après avoir rappelé brièvement le cas, indiquait la manière dont il devait être juridiquement résolu ${ }^{5}$. Ce recours au chef suprême de l'Église s'avérait d'autant plus indispensable que le Décret de Gratien qui avait recueilli, au milieu du XII ${ }^{e}$ siècle, l'héritage canonique antérieur, ne contenait guère de dispositions à ce sujet ${ }^{6}$. Dès la fin du XII ${ }^{e}$ siècle des recueils contenant les décrétales les plus typiques sont mis en circulation. Rapidement certains d'entre eux, notamment celui qui avait été confectionné par Bernard de Pavie aux alentours de 1191-1192, servirent de base à un enseignement qui contribua, lui aussi, pour une part non négligeable, à l'élaboration de ce droit ${ }^{7}$.

Sous le titre Qui filii sint legitimi sont donc progressivement rassemblées des décrétales à partir desquelles les canonistes, qui sollicitent souvent les secours du droit romain en la matière (quitte à lui faire dire tout autre chose que ce qu'il dit...), vont dégager les principales règles concernant la légitimité.

Summa decretalium ${ }^{8}$. Le principe de base était facile à poser : est légitime celui qui est né d'un mariage légitimement contracté, lequel, si aucun empêchement dirimant n'existe, est tout à fait valablement contracté par le seul consentement des époux ${ }^{9}$, voire même par de simples fiançailles suivies de copula carnalis ${ }^{10}$. Quid si le mariage se révèle $a$ posteriori comme entaché de nullité en raison de l'existence d'un empêchement que les futurs époux ignoraient? La question s'est vite posée, à propos de l'empêchement de parenté, et elle a été aussi vite résolue par Alexandre III : les enfants nés ou conçus avant que la sentence de nullité ait été prononcée sont légitimes à condition que le mariage ait été publiquement contracté et sine contradictione ecclesiae ${ }^{11}$. C'est ce qu'on appellera la théorie du mariage putatif : elle repose sur la bonne foi des époux. Mais que se passerait-il si l'un des époux seulement était de bonne foi? Si par exemple l'un d'entre eux cachait à l'autre qu'il est déjà marié, chose relativement facile, je n'ose pas écrire fréquente, à cette époque ? Le conjoint « innocent » et les enfants devraient-ils en pâtir? Certes non, comme le décidera in favorem prolis Innocent III dans une décrétale de 1205 , à l'encontre de l'opinion, professée jusque-là par une partie de la doctrine ${ }^{12}$. Pour que la théorie du mariage putatif joue, il suffit que l'un des époux soit de bonne foi. Donc même issus d'un mariage dissous pour cause de nullité, les enfants pourront éventuellement être légitimes. Ils pourront l'être aussi si leurs parents obtiennent $a$ posteriori une dispense du pape, à condition, bien sûr, que l'empêchement soit de ceux dont on peut dispenser ${ }^{13}$. La dispense dans ce cas, par un effet rétroactif qui lui est spécifique, "guérira le mariage à la racine » (sanatio in radice)) et permettra ainsi aux enfants issus de l'union d'être tenus pour légitimes ${ }^{14}$.

7 À cette première interprétation de la légitimité, qui concerne les enfants nés ou conçus pendant un mariage, va s'en ajouter une seconde que le droit canonique médiéval a emprunté au droit romain de Justinien ${ }^{15}$. Il s'agit de la légitimation de l'enfant naturel par le mariage subséquent de ses parents. Elle sert, d'une certaine manière, de 
tempérament à la rigueur de la discipline matrimoniale que l'Église cherche alors à imposer, en même temps qu'elle exalte la force du mariage.

Introduite dans le droit canonique médiéval par deux décrétales d'Alexandre III : la décrétale Conquestus $(\mathrm{X}, 4,17,1)$ et la décrétale Tanta $(\mathrm{X}, 4,17,6)$ qui deviendra très vite le siège de la matière (sedes materiae), la légitimation par mariage subséquent concerne les enfants naturels simples, à l'exclusion des enfants adultérins et incestueux ${ }^{16}$. Peuvent bénéficier d'une légitimation par le mariage subséquent de leurs parents les enfants nés de parents qui auraient été libres de contracter mariage entre eux. À quel moment? Au moment de la conception ou au moment de la naissance ? La question soulevée par un grand canoniste, Hostiensis (Henri de Suse, $\uparrow 1271$ ), sera fort débattue ${ }^{17}$. Sous son influence et celle de Ioannes Andreae ( $†$ 1348), elle sera résolue dans un sens favorable à l'enfant puisqu'on estimera qu'il suffit que les parents aient été capables de contracter mariage à un moment quelconque entre la conception et la naissance, pour que l'enfant puisse être légitimé. Cela permettra notamment de légitimer un enfant conçu par des personnes parentes à un degré prohibé, à condition qu'elles aient obtenu, avant la naissance, une dispense pour se marier ${ }^{18}$.

9 À cette légitimation par mariage subséquent est venue s'ajouter, dès le pontificat d'Innocent III, la légitimation par rescrit. Inspirée, elle aussi du droit romain de Justinien, elle joue pour la première fois en faveur des enfants de Philippe-Auguste et d'Agnès de Méranie, mais soulève immédiatement un grave problème. À la demande du roi qui avait, peut-être, seulement sollicité la reconnaissance du caractère légitime des enfants issus de son mariage avec Agnès ${ }^{19}$, le pape répond par une légitimation ${ }^{20}$. Nous "décorons", écrit-il : praedictos puerum et puellam de speciali gratia legitimationis titulo ut nullus eis in naturalibus defectus obsistat. C'est donc une légitimation pleine et entière. Dans quelle mesure le pape a-t-il ainsi le droit de légitimer? Certes le souverain pontife, par l'intermédiaire des dispenses ex defectu natalium, exerçait depuis longtemps déjà le pouvoir de légitimer ad sacra afin que les enfants illégitimes puissent rentrer dans les ordres ${ }^{21}$, mais pouvait-il aller au delà ? Cette légitimation par rescrit que le droit romain réservait exclusivement à l'empereur, peut-elle être également exercée par le pape et avoir un effet in temporalibus ? N' est-ce point un empiètement caractérisé du pouvoir spirituel sur le pouvoir temporel?

10 Justifiée par le fait que «le roi de France n'a pas de supérieur au temporel ${ }^{22}$, l'intervention d'Innocent III paraît, à la lumière de la fameuse décrétale Per venerabilem qui la suit de peu, comme «extraordinaire »... Mais elle n'est pas sans équivoque car le pape avait semblé en faire précisément une extension de son droit de légitimer in spiritualibus $^{23}$, et s'il avait quelque peu modéré son propos l'année suivante dans la décrétale Per venerabilem, il y laissait entendre néanmoins qu'il pouvait intervenir occasionnellement certis causis inspectis ${ }^{24}$. On saisit immédiatement le caractère redoutable que prend alors, au nom de la précellence du spirituel sur le temporel ${ }^{25}$, ce pouvoir et les vives réticences qu'il engendrera seront à la mesure du péril.

11 En réalité, il n'avait pas fallu attendre la décrétale Per venerabilem pour que le pouvoir temporel prenne conscience du danger que représentait pour lui cette compétence de l'Église en matière de légitimité. Dès le pontificat d'Alexandre III, l'inquiétude est visible et se traduit tout d'abord par le souci de délimiter les compétences respectives des juridictions laïques et ecclésiastiques. Une affaire concernant l'Angleterre, affaire à propos de laquelle Henri II se dira fort troublé, va permettre de poser sous la forme d'un grand principe une première limite à la compétence de l'Église. Elle intervient peu 
de temps après le compromis d'Avranches (1172) et fait l'objet d'une décrétale insérée dans plusieurs collections dès la fin du XII ${ }^{\mathrm{e}}$ siècle et amplement commentée, la décrétale Causam que ${ }^{26}$. Un dénommé Robert avait été dépossédé, par son grand-oncle, de l'héritage lui venant de son grand-père maternel, au motif que sa mère ayant été une fille adultérine n'avait pu lui transmettre quelque droit que ce soit à l'héritage. La légitimité de la mère de Robert était donc en cause et devait être jugée par des légats pontificaux ${ }^{27}$ mais le pape avait ordonné qu'avant tout jugement, Robert fut restitué dans ses biens, s'il en avait été dépossédé par la violence ${ }^{28}$. C'est ce qui avait «ému et troublé » Henri II. Alexandre III reconnaît le bien fondé de cette émotion parce que c'est au roi et non à l'Église qu'il appartient de juger de ces questions de possession ${ }^{29}$. Il donne donc l'ordre à ses légats quatenus regi possessionum iudicium reliquentes de ne s'occuper strictement que de la question de la légitimité. Seule la détermination de la légitimité de la personne en cause relève de la compétence de l'Église. L'affaire successorale qui a amené la contestation de la légitimité est une affaire civile entre laïcs dont l'Église n'a pas à connaître. C'est au juge laïc qu'il appartient de tirer les conséquences de la sentence de légitimité ou d'illégitimité rendue par le juge ecclésiastique. En ordonnant à ses légats de réintégrer avant tout jugement Robert en ses possessions, Alexandre III avait dépassé les limites de son pouvoir tout comme il l'avait fait l'année précédente en ordonnant à Bartholomé d'Exester et Roger de Worcester d'user de censures pour protéger l'héritage d'une femme dont la légitimité devait être prononcée par eux ${ }^{30}$.

12 Ainsi admise par l'Église elle-même, même si c'est un peu contrainte et forcée, cette stricte répartition des compétences constitue une limite importante ${ }^{31}$. Il n'en reste pas moins que c'est l'Église qui, en fonction des règles qu'elle a fixées concernant le mariage, se prononce sur la légitimité. C'est une compétence qui ne lui est pas déniée tant qu'elle reste strictement dépendante des conditions de validité du mariage, et elle est universellement reçue y compris jusqu'en la théorie du mariage putatif. "Tuit sachent que li mariages qui es tenus pour bons par le tesmoignage de sainte Église, écrira Beaumanoir dans ses Coutumes de Beauvaisis (1283), ne peut estre debatus ne corrompus en court laie, ne li enfans qui en nessent tenu pour bastart, tout soit ce que sainte Église ait fait grâce a l'homme et a la fame a soufrir le mariage $»^{32}$.

Les choses changent quelque peu en ce qui concerne la légitimation par mariage subséquent. Même si celle-ci est justifiée par la force du mariage sacramentel, elle n'en apparaît pas moins comme une "extension» qui engendre une insécurité sur le plan successoral qu'accroît le caractère rétroactif que la doctrine canonique a donné à la légitimation ${ }^{33}$. L'importance que revêt alors, économiquement et politiquement, la transmission héréditaire du patrimoine donne à la question toute sa dimension. On comprend dès lors que l'Angleterre déjà réticente quant au rôle joué par l'Église en matière de légitimité ait, en s'opposant à l'admission de la légitimation par mariage subséquent, refusé de conformer le droit anglais au droit canonique, comme le lui demandait Robert Grosseteste, un de ses évêques les plus en vue au moment du concile de Merton. Ce sera le fameux «statut» de Merton (1236) pris à la demande unanime des barons $^{34}$. Moins spectaculairement, les réticences de l'Empire à l'égard de la légitimation par mariage subséquent, bien connues à propos des fiefs puisque les Libri feudorum dénient au légitimé le droit d'y succéder ${ }^{35}$, semblent avoir persisté, d'une manière générale jusqu'à la fin du Moyen Âge ${ }^{36}$. En revanche les auteurs coutumiers français témoignent, dès le XIII ${ }^{e}$ siècle, d'une très large réception de l'institution, tout 
comme divers statuts italiens. Le Livre des coutumes de Milan (1216), par exemple, contrairement aux Libri feudorum, admet le légitimé à succéder aux fiefs ${ }^{37}$. La légitimation par mariage subséquent est également reçue dans les Pays-Bas méridionaux ${ }^{38}$ ou encore en Espagne ${ }^{39}$.

L'attitude du pouvoir temporel à l'égard de la légitimation par mariage subséquent sera donc, à l'exception de l'Angleterre, relativement respectueuse du droit canonique, précisément parce que cette légitimation conserve un lien étroit avec le sacrement de mariage.

Il n'en ira plus du tout de même avec la légitimation par rescrit du pape. Le risque d'empiètement du spirituel sur le temporel, si l'on admettait que le pape puisse légitimer non seulement in spiritualibus mais également in temporalibus, était bien trop considérable. Les premiers canonistes à gloser la décrétale Per venerabilem en sont euxmêmes conscients et nombre d'entre eux se prononcent pour une séparation effective des pouvoirs en ce domaine: à l'Empereur le pouvoir de légitimer in temporalibus, au Pape celui de légitimer in spiritualibus ${ }^{40}$. En dehors de l'exception que constitue la légitimation des enfants de rois, le pape ne peut légitimer in temporalibus que dans les territoires qui sont soumis à sa juridiction temporelle ${ }^{41}$. Certes il y a bien quelques canonistes, comme Jean le Teutonique, qui tentent d'utiliser en faveur d'une extension du pouvoir du pape l'argument de la supériorité du spirituel sur le temporel. Encore n'en font-ils bien souvent qu'une conséquence indirecte de la légitimation in spiritualibus ${ }^{42}$. Telle est en particulier la position d'Innocent IV qui, si convaincu qu'il soit de la supériorité du spirituel sur le temporel, ne se montre pas moins d'une extrême prudence, en écartant dans ce cas tout effet successoral ${ }^{43}$.

Une telle extension était en effet inadmissible pour les détenteurs du pouvoir temporel et dans les Siete partidas du roi Alfonse le Sage, par exemple, il sera bien précisé que la dispense pour entrer dans les ordres «no puede dispensar con ellos quanto en las cosas temporales $»^{44}$. Elle était difficilement justifiable dans la mesure où cette légitimation n'avait plus aucun lien obligatoire avec le mariage, alors que c'était ce dernier qui, nous l'avons vu, était à la source de la compétence de l'église en matière de légitimité. Ce lien étroit sera rappelé par Hostiensis qui fait du pouvoir de légitimer du pape une conséquence directe de son pouvoir de légiférer en matière de mariage ${ }^{45}$.

Conscient des vives réticences que la légitimation par rescrit rencontrait auprès des détenteurs $\mathrm{du}$ pouvoir temporel, Ioannes Andreae va reprendre cet argument fondamental mais pour limiter le champ d'application de la légitimation par rescrit. Dans son commentaire de la décrétale Per venerabilem, il subordonnera l'efficacité temporelle de l'intervention du pape au cas où un mariage nul était à l'origine de la naissance ${ }^{46}$. La légitimation par rescrit ne pourra avoir d'effet in temporalibus que là où, par une dispense, le pape rend rétroactivement valide le mariage qui était nul ab initio en raison de l'existence d'un empêchement dirimant. C'est un droit qui lui appartient incontestablement et dont il use depuis longtemps déjà, lorsque écrit Ioannes Andreae ${ }^{47}$. En validant ainsi rétroactivement le mariage nul, le souverain pontife légitime les enfants qui en sont issus. En dehors des états pontificaux, la légitimation par rescrit est ainsi restreinte par Ioannes Andreae, qui sera suivi par presque tous les canonistes ultérieurs, à l'ancienne pratique de la dispensatio. On parlera de dispensatio in radice ou encore, beaucoup plus tardivement, de sanatio in radice ${ }^{48}$.

18 Même ainsi circonscrite, la compétence de l'Église restait néanmoins une gêne pour le pouvoir temporel. L'obligation pour le juge séculier de renvoyer la question de la 
légitimité au juge d'Église et de surseoir à statuer en attendant sa décision pouvait apparaître comme une perte de temps préjudiciable au bon fonctionnement de la justice... Mais plus fondamentalement, c'est l'intervention même de la justice ecclésiastique qui est de plus en plus mal supportée. Le conflit qui en Angleterre aboutit au Statut de Merton a pour conséquence de priver la justice ecclésiastique de toute compétence lorsque la question surgit au cours d'un procès par «assise $»^{49}$. D'une manière moins radicale, le juge laïc va, dans d'autres pays et notamment en France, réussir à éliminer progressivement ce renvoi au juge d'Église en utilisant un argument de procédure. Cet argument, lui-même d'ailleurs emprunté à la procédure romanocanonique, permet de considérer l'illégitimité soulevée par l'une des parties comme une exception dont le juge saisi du principal peut connaître lorsque l'on agit au possessoire ${ }^{50}$. Or la plupart des procès, lorsque la propriété d'immeubles est en jeu, sont menés au possessoire avant de l'être au pétitoire. Les tribunaux ecclésiastiques vont donc ainsi progressivement perdre leur compétence, la question de la légitimité étant jugée de manière incidente par le juge saisi du principal. Beaumanoir témoigne que c'est chose faite à la fin du XIII dans le comté de Clermont, lorsque sont en cause des " eritages qui sont tenu de fief lai " $^{51}$. Mais il témoigne aussi, et bien d'autres après lui, de l'application qui sera faite par le juge laïc, en cette circonstance, des règles dégagées par le droit canonique.

Les juridictions ecclésiastiques ont été ainsi dépossédées de leur compétence mais le droit canonique a conservé son emprise. Pour l'histoire du droit et celle des relations entre Droit et Pouvoir, c'est une donnée non négligeable.

\section{NOTES}

1.Décrétales de Grégoire IX (recueil désigné ci-après sous son sigle traditionnel : $\mathrm{X}$ ), 2 , 10, 3. Cette décrétale est liée à la question de la succession au comté de Champagne. L'édition utilisée est celle d'E. Friedberg, Liepzig, 1879 (réimp. Graz, 1955).

2.Née à la fin du VI e siècle, renforcée à l'époque carolingienne par l'étroite collaboration entre les deux pouvoirs, la compétence de l'Église s'affirme comme exclusive à la fin du XI siècle. Mais ce n'est que progressivement que les normes qu'elle précise sur le plan théologique et juridique tout au long du XII ${ }^{\mathrm{e}}$ siècle s'imposeront. À cet égard les pontificats d'Alexandre III et, encore plus, d'Innocent III marqueront une étape décisive. Sur l'établissement de cette compétence, cf. notre Introduction historique au droit des personnes et de la famille, Paris, PUF (Droit fondamental), 1996, n 90-91 et 94 où l'on trouvera une bibliographie sur cette question.

3.Très significatif sera à cet égard le commentaire de Sinibaldo Fieschi, devenu le pape Innocent IV sur la décrétale Lator (X, 4, 17, 5) : Nota, écrira-t-il vers 1251, quod objiciens illegitimitatem, eam probare debet et per consequens non est matrimonium inter parentes... (Commentaria, éd. Francfort, 1570, fol. 479).

4.Inspirée des rescrits impériaux, la pratique des décrétales est apparue de bonne heure dans l'Église; mais si l'on a des témoignages de ce recours à l'autorité romaine 
dès le II ${ }^{e}$ siècle, la plus ancienne décrétale conservée dans les collections canoniques ne date que de la fin du IV e s. Cf. J. Gaudemet, Église et Cité, Paris 1994, p. 46sq. Sur la renaissance du pouvoir législatif pontifical, cf. H.Vidal, «Le Pape législateur de Grégoire VII à Grégoire IX ", Renaissance du pouvoir législatif et genèse de l'État, Montpellier 1988, p. 261sq.

5.Le terme décrétale (epistula decretalis) vient du latin decernere= décréter. La manière de résoudre le cas s'impose donc à l'évêque concerné mais aussi dans un cas semblable à tout autre évêque. Les décrétales forment avec les canons conciliaires l'une des deux grandes sources du droit canonique.

6.Quelques textes épars parmi lesquels le plus souvent cité est le c. Dicat (C. 32, q.4, c. 9).

7.Le recueil de Bernard de Pavie, appelé Compilatio prima, contient essentiellement des décrétales d'Alexandre III. Le plan adopté par Bernard: division en 5 livres eux-mêmes subdivisés en titres rassemblant diverses décrétales traitant du même sujet, sera celui de toutes les compilations ultérieures. On le retrouve notamment dans la Compilatio tertia (1209-1210) de Pierre de Bénévent, contenant pour l'essentiel des décrétales d'Innocent III, et surtout dans les Décrétales de Grégoire IX, compilation officielle faite sur l'ordre de ce pape par Raymond de Peñafort, et promulguée en 1234.

8.L. 4, t. 18. Cette Summa écrite entre 1193 et 1198 par Bernard résumant ainsi son propre recueil, a été très utilisée, notamment en France. Éd. A. Laspeyres, Regensburg, 1860 (réimp. Graz, 1956).

9.Les mariages clandestins sont valides même s'ils sont réprouvés. Innocent III par une disposition prise dans le cadre du IV e concile de Latran (1215), proclamera illégitimes les enfants issus de mariages clandestins contractés entre des personnes parentes à un degré prohibé (qui vient d'être ramené au $4^{e}$ degré par ce même concile), même si les conjoints ignoraient l'empêchement. La doctrine s'ingéniera à restreindre la portée de cette mesure.

10.Décrétale Per tuas: $(\mathrm{X}, 4,17,12)$. Le mariage peut se former en effet par paroles de présent (per verba de presenti) ou par fiançailles suivies d'union charnelle (per verba de futuro, carnali copula subsecuta).

11.Décrétale Cum inter $(\mathrm{X}, 4,17,2)$.

12.Décrétale Ex tenore $(\mathrm{X}, 4,17,14)$. Huguccio $(\dagger 1210)$ qui fut un canoniste de grand poids, et peut-être le maître d'Innocent III, s'était prononcé dans sa Summa sur le Décret de Gratien (écrite vers 1188-1190) pour un "partage »: l'enfant aurait été légitime à l'égard du parent de bonne foi et illégitime à l'égard de l'autre!

13.Le droit de dispenser est le corollaire du droit de légiférer mais ne peut aller à l'encontre des empêchements de droit divin. Sur ces dispenses a posteriori avant le IV ${ }^{e}$ concile de Latran (1215) et les embarras de la doctrine canonique à leur sujet, cf. l'étude de F. Demoulin, « Exceptio temporis : prescription romaine et dispense en matière matrimoniale au début du XIII ${ }^{e}$ siècle ", Proceedings of the Tenth International Congress of Medieval Canon Law, Rome, Biblioteca Apostolica Vaticana (Monumenta iuris canonici, series $C$, t.11), sous presse.

14.L'expression sanatio in radice est tardive alors que la pratique de la dispense $a$ posteriori validant un mariage antérieur et légitimant rétroactivement les enfants ne l'est pas. Sur les liens de cette sanatio avec la légitimation par rescrit du pape, cf. infra. 15.Justinien, empereur d'Orient (527-565) est à l'origine de vastes compilations de droit

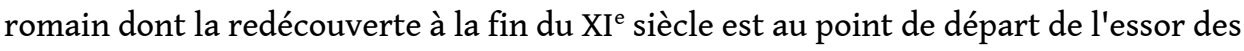


sciences juridiques en Europe. Sur cette renaissance cf. E. Cortese, Il rinascimento giuridico medievale, $2^{\mathrm{e}}$ éd., Rome, 1996. Justinien a joué un rôle fondamental dans la création de la légitimation par mariage subséquent. Ce sont ses constitutions qui servent de référence aux canonistes qui ne se privent pas néanmoins de les interpréter très librement.

16.Ils sont qualifiés de spurii. Figurent également parmi les spurii les enfants issus de rapports avec une personne tenue au célibat ecclésiastique ou liée par un vœu de chasteté.

17.Sur ce débat qui conduira également à définir l'enfant légitime comme l'enfant né dans le mariage, cf. notre étude «Tanta est vis matrimonii : remarques sur la légitimation par mariage subséquent de l'enfant adultérin », Studia Gratiana, vol. XXIX, 1998, p. 543sq.

18.Sur l'adoption par le droit français de cette interprétation, cf. notre étude : «Tanta est vis matrimonii : l'écho français d'une vieille controverse", Hommage à Romuald Szramkiewicz, Paris, Litec, 1998, p. 95sq.

19.C'est en effet le même terme legitimatio qui est employé à l'époque pour désigner la légitimité et la légitimation. Le terme legitimitas= légitimité existe mais il est très rarement employé dans notre domaine avant le $\mathrm{XIV}^{\mathrm{e}} \mathrm{s}$. Philippe avait épousé Agnès en 1196, alors que l'affaire de l'annulation de son mariage avec Ingeburge de Danemark n'était pas définitivement résolue, Ingeburge ayant fait appel au Pape (sur cette affaire, cf. J.Gaudemet, «Le dossier canonique du mariage de Philippe Auguste et d'Ingeburge de Danemark (1193-1213) ", Revue historique de droit français et étranger, 1984, p. 15sq). Les deux enfants étaient nés en 1198 (Marie) et en 1200 (Philippe) et Agnès venait de mourir (juillet 1201). Nous ne possédons pas le texte de la demande adressée par Philippe-Auguste à Innocent III, mais il est possible que le roi ait essayé de profiter des circonstances, notamment politiques, pour tenter de forcer la main au pape afin de faire jouer en faveur de ses enfants les effets d'un mariage putatif. Le passage de la lettre d'Innocent cité ci-dessous pourrait le laisser penser, tout comme la fin d'une lettre de Philippe-Auguste à Innocent à propos de son attitude lors du procès de Soissons, cf. R.Genestal, Histoire de la légitimation des enfants naturels en droit canonique, Paris, 1905, p.197.

20.Lettre du 2 novembre 1201 à Philippe-Auguste et lettre du même jour adressée à l'épiscopat français (Apostolica sedes, Patr. lat., t. 214, col.1191-1194). Dans cette lettre Innocent feint de croire en une certaine bonne foi du roi en la licéité de son mariage avec Agnès. Il y écrit en effet que Philippe a affirmé vehementer qu'après l'annulation (13 mars 1195) pour vice de forme de la première sentence : nulla tamen ad ipsum de alia non ducenda prohibitio facta pervenit et propter probationes affinitatis exhibitas coram eodem archiepiscopo quas idem rex veras esse credebat, inter eum ac praefatam nobilem putabat esse vinculum conjugale, quamquam nostra fuerit auctoritate compulsus ut et ipsam dimitteret et reginam reciperet memoratam. Sur la parenté entre Ingeburge et la première femme de Philippe-Auguste, cf. M.-B. Bruguière, «Le mariage de Philippe-Auguste et d'Isambour de Danemark : aspects canoniques et politiques », Mélanges offerts à Jean Dauvillier, Toulouse 1979, p. 135sq.

21.Sur cette légitimation ad sacra, cf. R. Génestal, op. cit., p. 43sq.

22.Le pape affirme en effet qu'il peut intervenir presertim ad petitionem eorum qui praeter Romanorum pontificem alium inter homines superiorem minime recognoscunt (P. L. 214, col.1193). La justification sera reprise avec encore plus de force dans la décrétale Per 
venerabilem $(\mathrm{X}, 4,17,13)$ : quum rex ipse superiorem in temporalibus minime recognoscat, décrétale qui date très probablement de début décembre 1202 ; elle répond à la demande de légitimation de ses enfants que lui avait adressée Guillaume de Montpellier. Sur cette demande, cf. H.Vidal, «Aspects montpellierains de la bulle Per venerabilem ", Revue de la Société d'histoire du droit des pays de droit écrit, 15, 1991, p. 49sq. 23.P. L. 214, col.1193: Cum igitur major idoneitas in spiritualibus quam in saecularibus requiratur, dubitari non debet quin ipsa tales ad actus legitimare valeat saeculares...

24. Rationibus igitur his inductis regi gratiam fecimus requisiti, causam tam ex veteri quam ex novo Testamento trahentes quod non solum in ecclesiae patrimonio (super quo plenam temporalibus gerimus potestatem) verumetiam in aliis regionibus, certis causis inspectis, temporalem iurisdictionem casualiter exercemus (X, 4, 17, 13, éd. Friedberg, col.716). Sur le caractère équivoque de la décrétale Per venerabilem à ce sujet, cf. B. Tierney : «Tria quippe distinguit iudicia... A note on Innocent III's decretal Per venerabilem ", Speculum, 1962, p. 48sq.

25. C'est à juste titre que M.Boulet-Sautel soulignait qu'il ne s'agissait pas ici d'une quelconque confusion entre spirituel et temporel, mais bien d'une supériorité affirmée du premier sur le second, comme l'illustrait le passage suivant de Per venerabilem :

Videretur siquidem monstruosum ut qui legitimus ad spirituales fieret actiones, circa saeculares actus illegitimum remaneret. Unde quum in spiritualibus dispensetur, consequenter intelligitur in temporalibus dispensatum. M.Boulet-Sautel : "Encore la bulle Per venerabilem ", Studia Gratiana, 13, 1967, p. 371sq.

26.Cette décrétale qui date de 1178 figure dans plusieurs collections anglaises dont l'Appendix au III ${ }^{\mathrm{e}}$ concile de Latran et dans la Compilatio prima de Bernard de Pavie avant d'être reprise dans les Décrétales de Grégoire IX (X, 4, 17, 4 et 7). Sur la place du droit canonique en Angleterre aux lendemains du Compromis d'Avranches qui fait suite à la terrible affaire du meurtre de Thomas Becket, cf.M. Cheney, "The Compromise of Avranches of 1172 and the spread of Canon Law in England ", The English Historical Review, 56, 1941, p. 177sq. Le litige successoral à l'origine de la décrétale Causam que a été reconstitué par C. et A. Duggan, «Ralph de Diceto, Henry II and Becket », Authority and Power.Studies on medieval law and Governement presented to Walter Ullmann, ed. by B. Tierney and P. Linehan, Cambridge, 1980, p. 75sq. D'une manière plus générale sur les rapports entre les deux pouvoirs, C.R. Cheney, From Becket to Langton, Manchester, 1956. 27. Ce sont les évêques de Londres et de Winchester qui avaient été nommés au départ mais l'évêque de Londres étant le seigneur de Robert, et donc suspect aux yeux du grand oncle, fut remplacé par l'abbé d'Herford (X, 1, 29, 17).

28.Ce n'était que l'application de la règle canonique selon laquelle le spolié doit être avant tout restitué (spoliatus ante omnia restituatur, $\mathrm{X}, 2,13,7$ ), règle qui est à l'origine de la réintégrande.

29. Nos attendentes quod ad regem pertinet non ad ecclesiam de talibus possessionibus iudicare...

30.Décrétale Conquestus (X, 4, 17, 1) qui date de juillet 1177, cf. M. Cheney, op. cit., p.189. 31.Cette décrétale Causam que est souvent l'occasion pour les premiers décrétalistes de développer leurs idées à propos des rapports entre les pouvoirs temporel et spirituel, en général plutôt favorables à leur indépendance. Tel est en particulier le cas de Richard l'Anglais qui a enseigné à Bologne à l'extrême fin du XII ${ }^{\mathrm{e}}$ siècle : magister Huguccio dicit et bene quod a Deo solo imperator habet potestatem in temporalibus et papa in spiritualibus et sic distincta est ultimis dictis; prius est enim imperator qui coronam accipiat a 
papa vel gladium ab altari, ut di. xciii, Legimus [= Décret de Gratien, d.93, c. 24], antea fuit imperium quam apostolatus et ante fuit imperator quam apostolicus (Reims, Bibl. Mun., ms. 692, fol. 93 ${ }^{\mathrm{rb}}$ ). Ce commentaire sera repris et accentué quelques années plus tard par Bernard de Compostelle l'Ancien (Modène, Bibl. Estense, ms. a 4 R 16, fol. $58^{\mathrm{vb}}$ ). 32.Éd. A. Salmon, Paris, 1899, nº 586.

33.Sur les raisons qui sont à l'origine de cette rétroactivité qui n'existait pas en droit romain, cf. notre étude : «De la rétroactivité à la fiction : notes sur la légitimation par mariage subséquent en droit canonique », Studi in onore di Ennio Cortese, Rome, Il cigno Galileo Galilei, sous presse.

34.Dont F. Pollock et W. Maitland, The history of English law before the time of Edward I, 2 éd., Cambridge, 1898, t. 11, pp. 188-189, soulignaient l'absence de texte authentique pour cette fameuse résolution. Sur la compétence juridictionnelle de l'Église, cf. d'une manière plus générale, R.H. Helmholz, «Bastardy litigation in medieval England », The american journal of legal history, 1969, p. 360sq.

35.Libri feudorum, 2, 26, 4. La question a été néanmoins fort discutée par les juristes, sous l'influence notamment de Jacques d'Ardizzone, Jean Blanc et surtout de Jacques de Belvisio dont la glose sur les Libri feudorum a été écrite avant 1309, cf. G. Giordanengo, Le droit féodal dans les pays de droit écrit, Rome, École française, 1988, p.130. Le texte sera interprété comme visant seulement la légitimation par rescrit. Cf. B. Pitzorno, La legitimazzione nella storia delle istituzioni familiari del medio evo, Sassari, 1904, p. 244sq. 36.D'après F. Kogler : «Beïtrage zur Geschichte der Rezeption und der Symbolik der legitimatio per subsequens matrimonium ", Zeitschrift des Savigny-Stiftung, Germanistische Abteilung, 1904, p. 121, le droit coutumier allemand ignore encore au début du XIV siècle la légitimation par mariage subséquent. Sur l'évolution ultérieure, ibid., p. 122sq. Pour les pays romands, J.F.Poudret, Coutumes et coutumiers. Histoire comparative des droits des pays romands du XIII à la fin du XVI siècle, Berne 1998, t. II, p. 12 ; sur le témoignage d'une réception tardive, cf. les textes cités par L. Favre, La condition des enfants légitimes dans les pays romands au Moyen-Âge, Lausanne (Bibliothèque historique vaudoise, t. 82), 1986. Mais peut-être y a-t-il ici un problème de sources.

37.Titre 29, éd. F. Berlan, 1868. Sur les statuts italiens, B. Pitzorno, op. cit, . p. 255sq. 38.Ph.Godding, Le droit privé dans les Pays-Bas méridionaux du XII ${ }^{e}$ au XVIII ${ }^{e}$ siècle, Bruxelles, Palais des académies, 1987, pp. 114-115.

39.Cf. «Las siete partidas », pars IV, t. 13, c. 1.1, éd. de la Real Academia de la Historia, Madrid 1807, t. III, p. 84 ; Castillo, Utilis et aurea glosa super Leyes de Tauri, Salamantina, 1544, fol. LXIIII" , et A.Gomez, Ad leges Tauri commentarius, Cologne, 1628, fol. 90. 40.Tel est en particulier le cas, avec des nuances, de Laurent d'Espagne, de Vincent d'Espagne et de Tancrède. Sur les différentes interprétations des canonistes, outre l'ouvrage de R. Génestal cité note 19 , on pourra consulter le récent article de D. Courtney-Batson, "Per venerabilem : from practical necessity to judicial supremacy ", Pope Innocent III and his World, éd. J. C. Moore, Aldershot, Ashgate, 1999, p. 287sq., étant entendu néanmoins que l'auteur, ignorant l'article de G.Post, «The so-called Laurentius' apparatus to the Decretals of Innocent III in Compilatio III », The Jurist 1942, p. 5sq., attribue à Laurent d'Espagne l'apparat Servus appelatur contenu dans le ms. Paris, BN lat. 15398 (le texte qu'il cite note 34 dans lequel Laurent est opposé à Jean aurait pourtant dû éveiller son attention). 
41.Cf. R. Génestal, op. cit., p. 206sq. L'auteur cite à juste titre quelques exemples d'intervention pontificale hors des territoires soumis à sa juridiction, notamment lors du pontificat d'Innocent IV. Elles correspondent bien à la réserve faite par Innocent III d'une intervention casualiter, certis causis inspectis (supra, n. 24).

42. Ad hoc dic quod papa non habet potestatem legitimandi in temporalibus, set tamen eo ipso quod quis est legitimatus in spiritualibus, intelligitur legitimatus in temporalibus, unde per quamdam consequentiam legitimat set non directe, glose sur Per venerabilem (Comp. III ${ }^{\mathrm{a}}, 4$, 12, 2), sur le mot potestatem, éd. K. Pennington, A study of Johannes Teutonicus's Theories of Church Governement and of the Relationschip between Church and State, with an Edition of his Apparatus to Compilatio tertia, Ph. D. Dissertation, Cornell, 1972, t. II, pp. 628-629.

43.Le légitimé in spiritualibus l'est également quoad actus legitimos c'est-à-dire : quod possit esse iudex et testis, in paterna autem hereditate vel aliis non intelligatur dispensatum (In $V$ libros Decretalium commentaria, Francfort, 1570, sur Per venerabilem, sur le mot evaderet). Innocent IV reprend ici une idée déjà exprimée, en particulier par Vincent d'Espagne, cf. sa glose citée par D. Courtney-Batson, op. cit. n. 22 in fine.

44.Pars IV, t. 15, c. 4. Le même passage se fait l'écho d'une règle inverse bien connue des canonistes, à savoir que toute légitimation par rescrit de l'empereur ou du roi, n'a pas d'effet in spiritualibus.

45.Lectura in quinque libros Decretalium, Venise 1581, sur Per venerabilem, $\mathrm{n}^{\circ} 8$ in fine. Hostiensis en conclut que seul le pape a un véritable pouvoir de légitimer, car l'empereur n'a pas le pouvoir de légiférer en matière de mariage et donc pas celui de légitimer, mais seulement de dispenser, ibid., $\mathrm{n}^{\circ} 9$.

46.In quinque Decretalium libros Novella commentaria, Venise, 1581, sur Per venerabilem, $\mathrm{n}^{\circ}$ 30: Cum enim papa non possit facere vel supplere matrimonialem consensum ubi non fuit, ergo nec talem prolem legitimare poterit, nisi quoad suum forum... Si vero proles genita fuit ex coitu matrimoniali, licet de facto tamen contracto tunc distingue... si vero matrimonium esse non poterat quia impediente lege canonica... subdistingue quia aut papa utitur verbo legitimationis, legitimans matrimonium et prolem et tunc quoad utrumque forum sit proles legitima cum impedimentum a lege canonica inductum papa tollere potuerit... aut simpliciter utitur verbo dispensationis et tunc solum ad illa prohibita admittetur in quibus est dispensatum, cum tales dispensationes sunt odiosae.

47.Cf. supra, p. 96.

48. Ce dernier terme ne fait son apparition qu'au cours du XVIII ${ }^{\mathrm{e}}$ siècle, d'après J. Harrigan, The radical sanatio of invalid marriages. An historical synopsis and commentary, Diss. Washington, 1936, p. 36.

49.Cf. R. Helmholz, op.cit., p. 194sq. Néanmoins comme le démontre l'auteur les tribunaux ecclésiastiques conservent une certaine activité en la matière.

50.Ainsi qu'en témoigne, à la fin du XIII ${ }^{\mathrm{e}}$ siècle, Guillaume Durand dans son Speculum Iudiciale, Francfort, 1668, t. II, p. 202. Après avoir souligné la nécessité du renvoi lorsqu'on agit au pétitoire, il ajoute: Si vero agitur possessorie et reus objiciat prejudicialem, secus est.

51.Beaumanoir, Coutumes de Beauvaisis, op.cit., $\mathrm{n}^{\circ} 578$ : «Et pour ce que teus debas depent de l'eritage, convient il a la fois que juges seculiers s'entremete de connoistre la bastardie qui est proposee devant li ». 


\section{AUTEUR}

ANNE LEFEBVRE-TEILLARD

Université de Paris II 